\title{
Systemic treatments for hepatocellular carcinoma: challenges and future perspectives
}

\author{
Francesco Tovoli ${ }^{1}$, Giulia Negrini ${ }^{1}$, Francesca Benevento ${ }^{1}$, Chiara Faggiano ${ }^{1}$, Elisabetta \\ Goio ${ }^{1}$ \& Alessandro Granito*,1 \\ ${ }^{1}$ Department of Medical \& Surgical Sciences, University of Bologna, S Orsola-Malpighi Hospital, Bologna, Italy \\ *Author for correspondence: Tel.: +39 051214 2717; Fax: +39 051214 2725; alessandro.granito@unibo.it
}

Sorafenib has been the only approved systemic treatment of hepatocellular carcinoma (HCC) for almost a decade. Recently, two new drugs showed positive results in two Phase III studies. The RESORCE trial identified regorafenib as a valid second-line treatment for patients progressing to sorafenib, the REFLECT trial showed that lenvatinib is noninferior to sorafenib as front-line treatment. Following these trials, the therapeutic scenario will be dominated by anti-VEGFR drugs, with three different molecules showing a proven anticancer activity. Some open problems still remain and different immunotherapy trials are underway, following promising preliminary results. In this review we analyze: the most recent advancements about patients treated with sorafenib; the results of RESORCE and REFLECT trials; and the ongoing Phase III clinical trials. Finally, we discuss how they could address the current problems and possibly reshape the future of the systemic treatments for HCC.

First draft submitted: 4 September 2017; Accepted for publication: 16 January 2018; Published online: 8 February 2018

Keywords: cabozantinib • hepatocellular carcinoma • immunotherapy • lenvatinib • metronomic capecitabine • regorafenib • sorafenib • systemic therapies

The identification of effective systemic treatments for hepatocellular carcinoma (HCC) has always been a difficult challenge in oncology. Conventional chemotherapies, including doxorubicin and gemcitabine, failed to improve the overall survival (OS) of these patients [1]. Only in 2008, sorafenib, a VEGFR-inhibitor, demonstrated its efficacy in treating unresectable HCC in two randomized-controlled trials (RCTs) $[2,3]$.

These results were followed by a period of enthusiasm in which the difficulties of the past seemed to be overcome. This period was soon dissipated by the failure of numerous RCTs with the consequent inability to find either a second-line treatment or a therapeutic alternative for patients intolerant to sorafenib (Table 1) [4-10].

After almost a decade of disappointing results, new positive results arrived in 2016 and 2017. Regorafenib, another VEGFR-inhibitor demonstrated its efficacy as a second-line treatment [11]. In the front-line setting, a noninferiority Phase III RCT comparing lenvatinib versus sorafenib reached its primary end point [12]. Besides, the encouraging preliminary results of immunotherapy trials seem to authorize a cautious enthusiasm for the possible novel and highly innovative treatments $[13,14]$.

The aims of this review are: to summarize the most important studies concerning sorafenib treatment published in the last years; critically analyze the results of the successful trial of the last year (regorafenib and lenvatinib); report ongoing Phase III studies, speculating on possible therapeutic innovations.

\section{Sorafenib \& next-to-be-approved treatments Sorafenib}

To date, sorafenib is the only systemic agent globally available for use in HCC. It is an orally active multitarget kinase inhibitor targeting different cell surface tyrosine kinases (e.g., VEGFR-1, $-2,-3$ and PDGFR- $\beta$ ) as well as the intracellular serine-threonine kinases Raf-1 and B-Raf. As such, sorafenib inhibits tumor cell proliferation and angiogenesis and promotes tumor cell apoptosis [15-17]. 


\begin{tabular}{|c|c|c|c|}
\hline Name of the trial & Study drug & Therapeutic line & Primary end point \\
\hline No name & Linifanib vs sorafenib & 1 & No difference in OS (HR: $1.046 ; 95 \%$ Cl: $0.896-1.221$ ) \\
\hline SEARCH & $\begin{array}{l}\text { Sorafenib + erlotinib vs } \\
\text { sorafenib + placebo }\end{array}$ & 1 & No difference in OS (HR: 0.929; 95\% Cl: $0.781-1.106 ; p=0.408$ ) \\
\hline No name & Sunitinib vs sorafenib & 1 & Significant inferiority in OS (HR: $1.30 ; 95 \% \mathrm{Cl}: 1.13-1.50 ; \mathrm{p}=0.014)$ \\
\hline BRISK-FL & Brivanib vs sorafenib & 1 & No difference in OS (HR: $1.06 ; 95 \% \mathrm{Cl}: 0.93-1.22$ ) \\
\hline BRISK-PS & Brivanib vs placebo & 2 & No difference in OS (HR: $0.89 ; 95 \% \mathrm{Cl}: 0.69-1.15 ; \mathrm{p}=0.33$ ) \\
\hline REACH & Ramucirumab vs placebo & 2 & No difference in OS (HR: $0.87 ; 95 \% \mathrm{Cl}: 0.72-1.05 ; \mathrm{p}=0.14$ ) \\
\hline EVOLVE-1 & Everolimus vs placebo & 2 & No difference in OS (HR: $1.05 ; 95 \% \mathrm{Cl}: 0.86-1.27 ; p=0.68$ ) \\
\hline POLARIS2009-001 & ADI-PEG20 vs placebo & 2 & No difference in OS (HR: $1.02 ; 95 \% \mathrm{Cl}: 0.85-1.23 ; \mathrm{p}=0.88$ ) \\
\hline METIV-HCC & Tivantinib vs placebo & 2 & No difference in OS (HR: $0.97 ; 95 \% \mathrm{Cl}: 0.75-1.25 ; \mathrm{p}=0.81$ ) \\
\hline JET-HCC & Tivantinib vs placebo & 2 & No difference in PFS (further data still not released) \\
\hline
\end{tabular}

HR: Hazard ratio; OS: Overall survival; PFS: Progression-free survival.

\begin{tabular}{|c|c|c|}
\hline Study & Dose reduction (\%) & Treatment discontinuation (\%) \\
\hline SHARP & 26 & 38 \\
\hline Asia-Pacific & 30.9 & 19.5 \\
\hline SOFIA & 54 & 44 \\
\hline GIDEON Child-Pugh A & 40 & 29 \\
\hline GIDEON Child-Pugh B & 29 & 40 \\
\hline INSIGHT & 58.6 & 15.5 \\
\hline
\end{tabular}

The antitumor activity of sorafenib in HCC was assessed in two large double-blind, placebo-controlled, Phase III RCTs: the SHARP and the Asia-Pacific trials $[2,3]$. Both trials demonstrated that sorafenib at the dose of $400 \mathrm{mg}$ twice-daily significantly improved OS in patients with HCC not amenable for loco-regional procedures, wellpreserved liver function (97\% Child-Pugh A) and Eastern Co-operative Oncology Group (ECOG) performance status $\leq 2$ (Table 2). Subsequent sub analyses of the SHARP trial showed that sorafenib was effective for the treatment of HCC regardless of HCC etiology, tumor burden, tumor stage (BCLC-B or C), performance status (Eastern Co-operative Oncology Group 0, 1 or 2) and prior treatment (curative therapy or chemoembolization) [18-20]. However, the survival benefit is greater in patients with disease confined to the liver (without extrahepatic spread), or in those with hepatitis $\mathrm{C}$ virus, or a lower neutrophil-to-lymphocyte ratio, an indicator of inflammation status, as recently demonstrated in a pooled exploratory analysis of the two registrative Phase III studies [19].

A series of real-line clinical trials performed in different geographical regions confirmed these favorable data [21-23]. In particular, the results of the GIDEON study [21], the largest real-life observational study involving 3371 patients treated with sorafenib across 39 different countries, confirmed a good efficacy and safety profile. Consequently, all of the current international guidelines unanimously recognize sorafenib as the standard treatment for Child-Pugh A patients who have been diagnosed with advanced HCC [24].

The benefit of sorafenib in the Child-Pugh B population, for which the underlying cirrhosis represent a competitive cause of death, is highly debated. Because sorafenib is metabolized primarily in the liver by cytochrome P450 3A4 and UGT1A9, a more compromised hepatic function might alter drug clearance and dose-related toxicity [25,26]. In a preceding Phase II trial enrolling 137 patients, 38 (28\%) were classified as CPT B. The drugrelated toxicity was similar between Child-Pugh A and Child-Pugh B patients however, the latter patients had a higher frequency of hyperbilirubinaemia ( 40 vs $18 \%$ ), encephalopathy (11 vs $2 \%$ ) and severe ascites (18 vs $11 \%$ ).

A comparison of the pharmacokinetic parameters showed no significant differences between Child-Pugh A and Child-Pugh B patients. Although this finding was then confirmed by a Japanese Phase I study, the patient numbers of these two studies were too small to allow a meaningful pharmacokinetic comparison between the Child-Pugh classes [26]. 
In the two registrative trials were almost exclusively included Child-pugh A patients, with only 3-5\% classed as CPT B patients [2,3].

As such most evidences rely on real-life studies showing a rate of adverse effects in Child-Pugh B patients quite similar to that of Child-Pugh A counterpart, but only a narrow survival benefit [21-23,27-29]. Consequently, some international guidelines (Asia-Pacific Association for the Study of the Liver, National Comprehensive Cancer Network and European Association for the Study of the Liver) suggest a possible use of sorafenib even in Child-Pugh $\mathrm{B}$ patients, but limited to patients with a Child-Pugh score of 7 with mild elevation of bilirubin serum concentration and without diuretic uncontrolled ascites. However, Child-Pugh B, as well as elderly patients, represents a fragile population more often requiring a reduced dosage and a stricter monitoring [30-32].

Sorafenib is not free from adverse effects (the most frequent being skin toxicity, diarrhea, arterial hypertension and fatigue), potentially leading to dose reduction or treatment discontinuation [2-3,30]. Interestingly, data from field-practice studies (SOFIA, INSIGHT and GIDEON studies) point out a little greater rate of dose reduction or treatment discontinuation due to adverse events, reflecting the presence of comorbid patients in the real world (Table 2) [21-23].

In the GIDEON study the overall frequency of adverse effects ( 84 vs $89 \%)$ and those considered to be drugrelated adverse events (69 vs 64\%) was generally consistent across the Child-Pugh A and Child-Pugh B patients, as well as the frequency of grade 3 and 4 adverse events. However, serious adverse events (60 vs 36\%) and treatment discontinuation ( 40 vs $29 \%$ ) due to adverse events were more common in Child-Pugh B patients than in ChildPugh A patients. The incidence of individual adverse events and drug-related adverse events did not differ between Child-Pugh A and Child-Pugh B patients, with the exception of hand-foot skin reaction, which occurred more frequently in Child-Pugh A patients (32 vs 17\%) [21].

In this setting, a topic of growing interest is the prognostic significance of adverse events. As a matter of fact, at the moment there is a lack of biomarkers or other plasma predictors of sorafenib efficacy [33,34]. As many studies have pointed out, HCC has a significant genetic heterogeneity that can lead to primary or acquired resistance to sorafenib [35]. Although several mechanisms involved in this process have been recognized, we are far from identifying reliable biomarkers predictive of sorafenib resistance [36]. Hence, research has looked for other markers such as clinical predictors. Indeed, some studies tried to assess the correlation between adverse event development and treatment outcome [37-41], based on the concept that the occurrence of adverse events may be related to the sorafenib mechanism of action inhibiting one or more drug molecular targets. Early dermatologic adverse events currently appear to have a more convincing predictor role, since in a prospective study their appearance was significantly correlated with longer OS and time to progression (TTP) compared with patients not experiencing these symptoms, and resulted also correlated with complete response [39,40]. These studies call for research to validate this prognostic correlation, but they also pose further questions on the optimal management of patients without these specific side effects to improve the cost-effectiveness of sorafenib [42].

It is also worth of note that a significant interpatient variability of sorafenib pharmacokinetic has been reported and the available data would suggest a relationship between increased cumulated sorafenib exposure and adverse events [43]. An additional source of inter-individual different toxicity profile could be related to the genetic polymorphisms in VEGF and VEGFR [44].

Immediately after its approval as front-line systemic treatment, different studies verified the suitability of sorafenib as adjuvant therapy. In particular the Phase III STORM trial enrolled 1114 patients treated with liver resection or percutenous ablation. Patients were randomized in a 1:1 fashion and received either sorafenib or placebo. The main end point was recurrence-free survival, which was found to be similar in the two groups (33.3 months in the sorafenib group vs 33.7 months in the placebo group; hazard ratio [HR]: 0.940; 95\% CI: $0.780-1.134$; $\mathrm{p}=0.26)[45]$.

Besides, a combination of locoregional and systemic treatments (chemoembolization + sorafenib) in patients with intermediate-stage HCC has been largely investigated in recent years [46-48]. Overall, the results suggest that this combination failed to result in meaningful benefits, showing longer time to progression, but without improving the OS [49].

Further, the results of a Phase I trial strongly discouraged a combined strategy of Stereotactic Body Radiation Therapy and concurrent use of sorafenib due to the high prevalence of severe adverse events, including two cases of ruptured HCC [50]. 
Currently, both the STOP-HCC trial [51] and a specifically dedicated cohort of the SORAMIC trial [52] are evaluating whether a combination of TARE and sorafenib may prolong the OS of patients with locally advanced HCC compared with sorafenib alone.

In absence of second-line treatments approved, the question whether sorafenib should be stopped on radiologic progression alone still remains a clinically relevant topic. In the SHARP trial the authors made clear that the statistical significance was obtained also thanks to the possibility to treat patients even beyond radiologic progression, provided that the investigator believed it was beneficial for the patient [2].

The benefit of treatment beyond radiological progression was confirmed by real-life studies, which also demonstrated that a tumor burst may occur at the withdrawal of sorafenib. The reasons for this rapid tumor spread are complex but probably involve the reactivation of the previously inhibited VEGFR pathway, together with the acquired activation of alternative pathways [53]. As such, while waiting the approval of second-line therapies for HCC progressors, sorafenib could still be offered beyond radiological, but not symptomatic progression. Once second-line treatments will be available, the most appropriate management after sorafenib failure will have to be investigated in future large trials [54].

The availability of regorafenib as a second-line therapy may modify this orientation and will lead to future studies to identify the best timing for a therapy switch.

Some information about this topic may be already deducted from two important studies. Reig et al. [55] described prospectively followed 147 HCC patients treated with sorafenib. Radiological response was assessed after 1 month and then every 8 weeks. Baseline BCLC C status, deterioration of liver function, compromised performance status, time to progression and permanent sorafenib withdrawal were all independently associated with a shortened OS. More interestingly, the authors described four different pattern of progression (intrahepatic increase in tumor size, extrahepatic increase in tumor size, new intrahepatic lesions, new extrahepatic lesions) and found that the latter pattern is also independently associated with a worse OS. As such, the authors concluded that not all pattern of progression are equal and that the BCLC classification should be refined at the time of progression to better predict the prognosis. Iavarone $e t$ al. analyzed 200 patients who permanently discontinued sorafenib and were potentially eligible for second-line treatments [56]. Performance status, macrovascular invasion and extrahepatic spread were shown to be prognosticators of OS, but the most important finding was that the reason for sorafenib discontinuation was an independent prognostic factor as well. In particular, discontinuation due to adverse effects was associated with the best prognosis.

The findings by Iavarone and Reig brought important considerations which are useful in clinical practice and also represent critical factors in the design of clinical trials [57].

Finally, it has been recently reported that some scores mainly based on biochemical parameters, could be useful to predict the clinical benefit of sorafenib treatment as well as to better select patients for second-line treatment after sorafenib failure [58-60]. However their use in clinical practice need validations studies.

\section{Lenvatinib}

Lenvatinib is an oral multikinase inhibitor targeting the VEGFR, FGFR and PDGFR, as well as the RET and KIT pathways [61]. As such, its biological action is similar but completely overlapping with that of sorafenib. Currently, lenvatinib is approved in differentiated thyroid cancers refractory to radio-iodine administration [62]. Further, a combination of lenvatinib and everolimus has been approved as a second-line treatment for advanced renal cell carcinoma [63].

The efficacy and the safety of lenvatinib as a front-line treatment for advanced HCC has been tested in the recently concluded REFLECT trial. This non-inferiority Phase III trial randomized 954 patients with unresectable HCC to lenvatinib (8 mg or $12 \mathrm{mg}$ once-daily based on body weight) or sorafenib at $400 \mathrm{mg}$ twice-daily [12].

The primary end point of a noninferior OS was met (13.6 vs 12.3 months; HR: 0.92; 95\% CI: 0.79-1.06), making REFLECT the first positive global Phase III trial compared with sorafenib in first-line treatment for HCC. The median time to progression was 8.9 months with lenvatinib and 3.7 months with sorafenib (HR: 0.63 ; $\mathrm{p}<0.00001)$. In addition, lenvatinib demonstrated a significantly higher overall response rate compared with sorafenib $(24 \%$ vs $9 \%$; odds ratio: 3.13 ; $\mathrm{p}<0.00001)$. A similar number of patients in both arms had treatmentemergent adverse events. Most common treatment-emergent adverse events in the lenvatinib arm were hypertension (42\%), diarrhea (39\%), decreased appetite (34\%), decreased weight (31\%) and fatigue (30\%). Median treatment duration was 5.7 months $(0-35)$ for lenvatinib and 3.7 months $(0.1-38.7)$ for sorafenib. Discontinuation due to adverse events occurred in $13 \%$ of lenvatinib-treated and $9 \%$ of sorafenib-treated patients. 
The analysis of secondary end points brought some elements worth of discussion. In particular, the discrepancy between the stark superiority of lenvatinib in terms of TTP (7.4 vs 3.7 months) and objective response rate (24 vs $9 \%$ ) on one hand, and the lack of a full superiority in terms of OS raises further doubts about the well-known limits of surrogate end points in the evaluation of systemic treatments for HCC. However, at the time of writing this review, results of the REFLECT trial are available only as conference presentation, major considerations will be therefore feasible only after publication of full data.

In conclusion, the successful REFLECT trial will lead to the availability of a new drug for the treatment of advanced HCC. Even if lenvatinib failed to reach a full superiority compared with sorafenib, the competition between companies may lead to more affordable costs of anti-angiogenic drugs for lower-income countries and therefore to a more widespread opportunity of care. However, once lenvatinib will be approved for treating HCC patients, criteria for the differential use of sorafenib and lenvatinib as first-line therapy will need to be established.

\section{Regorafenib}

Regorafenib is an oral multikinase inhibitor with a structure very similar to that of sorafenib except for the addition of a fluorine atom in the center phenyl ring which confers a distinct biochemical and pharmacologic profile [64]. Its molecular targets include VEGFR-2, VEGFR-3, Tie-2, PDGFR, FGFR-1 and the mutant oncogenic kinases KIT, RET and B-RAF [65].

Tie-2 is predominantly expressed on endothelial cells and is a crucial regulator of angiogenesis, indispensible for the maturation of immature vessels. Thus, thanks to the combined blockage of VEGFR2 and Tie-2 signaling, regorafenib potentially inhibits tumor neoangiogenesis more potently than agents blocking VEGF signaling alone [66].

Regorafenib is the first agent that has been shown a significant survival benefit when administered as second-line therapy after sorafenib failure. In the randomized, placebo-controlled Phase III international study (RESORCE trial), regorafenib significantly improved OS compared with placebo in HCC patients who had experienced radiologic progression during sorafenib therapy (HR: 0.63; 95\% CI: 0.50, 0.79; $\mathrm{p}<0.0001$ ) [67].

The RESORCE study was conducted at 152 centers in 21 countries, in North America, South America, Europe, Asia and Australia. The patients who were eligible for the trial had progressed under sorafenib and were able to tolerate at least $400 \mathrm{mg}$ of sorafenib daily for at least 20 of the last 28 days prior discontinuation. Patients were randomized 2:1 to supportive care plus either regorafenib $160 \mathrm{mg}$ once-daily or placebo. Treatment consisted of cycles of 3 weeks on the agent followed by a 1-week break and was continued until disease progression, death, or unacceptable toxicity. Patients had to be randomized within 10 weeks of their last sorafenib dose. After stratification for various factors, including age, extrahepatic disease, macrovascular invasion and underlying disease, patients treated with regorafenib still had a significantly better OS [11].

The safety profile was generally consistent with that reported for other gastrointestinal malignancies [68]. In particular, rates of grade $\geq 3$ adverse events were $79.7 \%$ with regorafenib and $58.5 \%$ with placebo. Most common grade $\geq 3$ adverse events included hypertension ( 15.2 vs $4.7 \%$ ), hand-foot skin reaction (12.6 vs $0.5 \%$ ), fatigue ( 9.1 vs $4.7 \%$ ) and diarrhea (3.2 vs $0 \%$ ). Rates of interruptions or dose modifications due to adverse events were $68 \%$ with regorafenib and $31 \%$ with placebo, while $25 \%$ of patients in the regorafenib group versus $19 \%$ of patients in the placebo group discontinued due to adverse events.

In a post hoc exploratory analysis, the occurrence of hand-foot skin reaction during treatment suggested that this adverse event may be a marker for drug activity. Overall survival in patients developing hand-foot skin reaction was significantly longer compared with those who did not experience it (14.1 vs 6.6 months; HR: 0.52 ; $95 \%$ CI: $0.40-0.67$ ), as was previously shown for sorafenib. [69]

Interestingly, in the updated analysis of the RESORCE trial, the assessment of outcomes for the treatment sequence of sorafenib plus regorafenib showed an OS of 26 months from the start of sorafenib treatment among patients who received the sorafenib/regorafenib sequential therapy (19.2 months in the placebo group) [70]. This important extension in OS suggests that sequential systemic treatments may further improve the prognosis of a subgroup of patients with advanced HCC who are likely characterized by an appropriate management of adverse events during first-line sorafenib therapy and adequate selection for second-line treatment.

The 26 month OS observed with the sequential sorafenib/regorafenib therapy is in fact comparable with the conventional prognosis of patients with intermediate stage after TACE [49]. 


\section{Cabozantinib}

Cabozantinib is an oral multikinase inhibitor targeting MET in addition to VEGFR2. It also inhibits many other receptor tyrosine kinases (such as RET, KIT, AXL and FLT3) that have been implicated in neoplastic pathobiology [71]. Cabozantinib is currently approved by the US FDA for the treatment of advanced renal cell carcinoma and of medullary thyroid cancer.

The rationale for a dual VEGFR/MET blockade is supported by the consolidated evidence that resistance to VEGFR-targeted therapies may arise from the upregulation of alternative proangiogenic and proinvasive signaling pathways, including the MET pathway [72-74].

These aspects have been confirmed in the specific case of HCC as well, as the rate of MET-high HCC increased from a pre-sorafenib value of $40 \%$ to an almost double value after progression to sorafenib in a biomarker study of the eventually failed tivantinib trial [75].

A role of cabozantinib has been investigated in a Phase II randomized-discontinuation study in advanced HCC patients who had received one prior therapy, showing promising results and an overall disease control rate of $68 \%$. The most common grade $3 / 4$ adverse events were diarrhea (17\%), palmar-planter erythrodysesthesia (15\%) and thrombocytopenia (10\%) [76].

Based on these data, the CELESTIAL Phase III trial (NCT01908426) compared cabozantinib with placebo in patients with HCC and Child-Pugh A liver function who received prior sorafenib and may have received up to two prior systemic cancer therapies, making it a second- to third-line clinical trial [77].

The enrollment was completed in September 2017 and on October 2017 Exelixis announced that the CELESTIAL trial met its primary end point showing a statistically significant and clinically meaningful improvement in OS of patients treated with cabozantinib compared with placebo in advanced HCC [78].

\section{Ongoing Phase III trials \\ Ramucirumab}

Ramucirumab is a recombinant IgG1 monoclonal antibody antagonizing and the VEGFR-2 [5]. The original Phase III REACH trial investigated the efficacy of the safety of this molecule as a second-line treatment for unresectable HCC. The most common grade 1 or 2 treatment-emergent adverse events were peripheral edema (36\%), ascites $(22 \%)$ and headache $(18 \%)$. Any grade events that occurred at a higher frequency in the ramucirumab group than the placebo group included bleeding or hemorrhage (32\%), which was primarily due to grade 1-2 epistaxis (14\%) and gingival bleeding (6\%); hypertension $(20 \%)$, proteinuria $(17 \%)$, liver injury or failure $(51 \%)$, and infusion-related reactions (7\%).

No benefit in OS was observed in the ramucirumab treatment arm. However, the survival analyses of patient with baseline alpha-fetoprotein (AFP) concentration $\geq 400 \mathrm{ng} / \mathrm{ml}$ (which had been predefined in the study plan) shows a meaningful effect of the study drug on OS (HR: 0.674; median OS: 7.8 vs 4.2 months; $p=0.006$ ) [5].

Unfortunately, no stratification according to AFP values at the time of inclusion had been included in the original design of the study. As such, the novel Phase III REACH-2 study (NCT02435433) will compare ramucirumab versus placebo in patients with $\mathrm{AFP}>400 \mathrm{ng} / \mathrm{ml}[79]$.

The enrollment is currently closed and results are expected in late 2018 or 2019.

\section{Immune checkpoint inhibitors}

The escape from immunological surveillance is one of the main mechanisms of tumor progression. It can be the consequence of distinct immune alterations, including defective antigen presentation, dysfunctions of effector and regulatory $\mathrm{T}$ cell, alterations in immune checkpoint molecules, disarray of cytokine profiles. Their identification has provided the main rationale for the development of immunotherapy in HCC [80].

Outstanding achievements with immune checkpoint inhibitors in different malignancies have been reported in the last years. The best known checkpoint inhibitors are nivolumab, an inhibitor of the lymphocytes PD1 receptor, and ipilimumab, an inhibitor of CTLA-4. Also, a different PD1 inhibitor (pembrolizumab) and the antagonists of PD-L1 durvalumab, avelumab and atezolizumab have been developed [81]. All of these drugs inhibit an immune checkpoint exploited by the tumor cells to protect themselves from the immune system recognition and destruction [81,82]. As a matter of fact, nivolumab and ipilimumab revolutionized the treatment of metastatic melanoma and non-small-cell lung carcinoma.

The first evidences supporting the rationale of immune checkpoint inhibitors in HCC came from an investigatorinitiated Phase II clinical trial of the CTLA-4 inhibitor tremelimumab [83]. In this study, Sangro and collaborators 
recruited $21 \mathrm{HCV}$-related HCC, 57\% in the advanced stage, 42.9\% with Child-Pugh class B and $23.8 \%$ previously treated with sorafenib. Patients were treated with tremelimumab until progression or unacceptable toxicity. Partial response rate and disease control rate were 17.6 and 76.4\%, respectively. Median TTP was 6.48 months (95\% CI: 3.95-9.14). A significant drop in viral load was observed and this antiviral effect was associated with an enhanced specific anti-HCV immune response.

Even if some transient grade 3-4 aminotransferase elevation developed, no toxicities requiring systemic steroid treatment were registered.

Recently, the effects of tremelimumab have been studied in combination with locoregional therapy in 32 HCC patients, with the aim to enhance the tremelimumab activity by inducing immunogenic tumor cell death and local Inflammation. Tremelimumab was administered with transarterial chemoembolization in seven patients with BCLC-B, with radiofrequency ablation in ten patients with BCLC-C, and in 11 patients with BCLC-C underwent cryoablation. Tumor response was assessed in the 19 patients with evaluable lesions outside of the ablation area and the partial response rate was $26 \%(5 / 19)$, while the disease control rate was $84 \%(16 / 19)$ [84].

Subsequently, a large Phase IB/II multicohort trial investigating the safety of nivolumab alone or associated with ipilimumab was announced and is currently ongoing [85].

As of August 2017, the CheckMate-040 study (NCT01658878) includes: two dose escalation/expansion cohorts (cohort 1 and 2); a cohort for a randomized 1:1 comparison versus sorafenib in the frontline setting (cohort 3); a combination therapy cohort with ipilimumab for patients progressing or intolerant to sorafenib (cohort 4); a cohort reserved to patients with Child-Pugh B HCC (cohort 5); and a combination therapy cohort with cabozantinib (with or without ipilimumab; cohort 6) [85,86].

Results from the first two cohorts are now available and encouraging. In a total sample of 262 patients, grade $3 / 4$ treatment-related adverse events occurred in $20 \%$. The objective response rate (ORR) was $20 \%$ ( $95 \%$ CI: $15-26)$ in 214 patients treated in the dose-expansion phase with a median duration of response of 9.9 months and a disease control rate of $64 \%$ (95\% CI: 58-71) [87].

Results from cohort 3, 4 and 5 are pending (closed enrollment), while cohort 6 is still active and recruiting both sorafenib-naive and pretreated patients.

These preliminary results were convincing enough to promote the global Phase III RCT of nivolumab versus sorafenib as first-line treatment in patients with advanced HCC (CheckMate-459: NCT02576509) [88].

In parallel, another Phase III RCT is comparing pembrolizumab versus best supportive care in a second-line setting [89].

\section{Metronomic capecitabine}

Metronomic regimens are progressively gaining interest and popularity in oncology. Metronomic protocols rely on the continuous administration of low doses of antineoplastic drugs, without prolonged drug-free breaks. The potential efficacy of metronomic chemotherapy depends on multiple factors, including: reduction of the therapeutic resistance of the tumor, inhibition of tumoral angiogenesis and activation of both innate and adaptive immune response [90,91].

Until now, cytotoxic drugs administered according to standard protocols did not demonstrate a clear OS benefit in HCC patients [1]. Data about metronomic regimens, however, are still scarce. Metronomic capecitabine (MC) was evaluated in three different studies, mainly in a second-line setting. All of these studies showed a low rate of adverse events and a good efficacy [92-95]. However, the lack of prospective RCTs does not allow a definite judgement of MC biological efficacy.

Such studies would require a large amount of independent funds, as capecitabine patent has expired. Answering these calls, the Italian Medicines Agency recently financed a cost-effectiveness, randomized, cross-over study comparing capecitabine versus sorafenib [96]. This trial will include multiple Italian centers and will provide interesting information about MC both in frontline and in second-line setting.

\section{Conclusion \& future perspective}

Systemic treatment of HCC is rapidly evolving after a decade of disappointment and, at present, three new drugs (i.e., regorafenib, lenvatinib, cabozantinib) have been shown to be effective in Phase III clinical trials (Table 3).

Sorafenib remains the standard-of-reference in HCC systemic therapy, as of today. This role derives from the lack of clearly superior drugs as first-line treatment, and from the wide availability of real-life clinical data. It is entirely possible that lenvatinib may become a competitor frontline therapy; however, due to the similar mechanism of 
Table 3. Phase III randomized, controlled, clinical trials who met primary end point for patients with unresectable hepatocellular carcinoma.

\begin{tabular}{|c|c|c|c|c|c|}
\hline Drug & Study (year) & Line & Study design & Median OS months $(95 \% \mathrm{Cl})$ & $\mathrm{HR}(95 \% \mathrm{Cl})$ \\
\hline \multirow[t]{6}{*}{ Sorafenib } & SHARP (2006) & 1 & Randomized, placebo-controlled & $10.7(9.4-13.3)$ & $0.69(0.55-0.87)$ \\
\hline & & & & vs & \\
\hline & & & & $7.9(6.8-9.1)$ & \\
\hline & Asia-Pacific (2007) & 1 & Randomized, placebo-controlled & $6.5(5.56-7.56)$ & $0.68(0.50-0.93)$ \\
\hline & & & & vs & \\
\hline & & & & $4.2(3.75-5.46)$ & \\
\hline \multirow[t]{3}{*}{ Regorafenib } & RESORCE (2016) & 2 & Randomized, placebo-controlled & $10.6(9.1-12.1)$ & $0.63(0.50-0.79)$ \\
\hline & & & & vs & \\
\hline & & & & $7.8(6.3-8.8)$ & \\
\hline Lenvatinib & REFLECT (2017) & 1 & $\begin{array}{l}\text { Randomized, non-inferiority, lenvatinib } \\
\text { vs sorafenib }\end{array}$ & $13.6(12.1-14.9)$ vs & $0.92(0.79-1.06)$ \\
\hline Cabozantinib & CELESTIAL (2017) & $2-3$ & Randomized, placebo-controlled & Publication of data is expecte & 2018 \\
\hline
\end{tabular}

action and toxicity profile, it is more likely that in the next future its role in the first-line setting will be challenged by immune checkpoint inhibitors or their combination.

In the second-line scenario, the treatment sequence of sorafenib plus regorafenib is associated to an important extension in overall survival making the prognosis of this subgroup of patients with advanced HCC similar to that of those with intermediate stage underwent TACE. After positive results of the CELESTIAL trial, cabozantinib could become a further therapeutic alternative for patients failing sorafenib, although, at present we do not know the potential benefit of the sequence of sorafenib/cabozantinib.

Over the next 5 years, the scenario will depend on the success or failure of the immunotherapy trials. If successful, these new drugs might have a relevant role both in the first- and second-line setting as they could be the optimal strategy for patients with contraindication or poor tolerability to multikinase inhibitors. Moreover, if confirmed that immunotherapy can be carried out independently of causes less liver toxicity, this approach could allow treatment of HCC even in patients with more compromised liver function.

Immunotherapy could also potentially extend its therapeutic indication to the adjuvant setting, given the promising preliminary results in this field (where, on the contrary, sorafenib has shown no benefit). However, all of these potential uses are subject to ongoing or future trials in order to demonstrate a survival benefit.

Finally, the identification of biomarkers that could predict response or resistance to treatments is ideally the best way to guide treatment decision and to identify the best timing for a therapy switch. For this purpose, collection of tumor histological samples and biobanking in clinical trials is essential.

Financial \& competing interests disclosure

A Granito is a consultant for Bayer. The authors have no other relevant affiliations or financial involvement with any organization or entity with a financial interest in or financial conflict with the subject matter or materials discussed in the manuscript apart from those disclosed.

No writing assistance was utilized in the production of this manuscript.

\section{Open access}

This work is licensed under the Creative Commons Attribution 4.0 License. To view a copy of this license, visit http://creativecomm ons.org/licenses/by/4.0/ 
Executive summary

- To date, sorafenib is the only systemic agent globally available for use in patients with hepatocellular carcinoma (HCC).

- The efficacy and safety of sorafenib were assessed in two large double-blind, placebo-controlled, Phase III randomized-controlled trials and confirmed in large real-life clinical trials.

- No biomarkers predictive of response to sorefenib are available. However, patients suffering from early dermatological adverse events seem to have a better prognosis.

- Sorafenib treatment may be of benefit even after radiological progression. With second-line treatments available at the horizon, future studies should identify the more appropriate timing for switching therapies.

- In the Phase III REFLECT trial, lenvatinib has shown to be non-inferior to sorafenib in a front-line setting.

- In the second-line setting, both regorafenib and cabozantinib have shown to be superior to best supportive care following sorafenib failure. The treatment sequence of sorafenib plus regorafenib showed an OS of 26 months in patients who received the sorafenib/regorafenib sequential therapy.

- Immune checkpoint inhibitors are attracting more and more attention, especially after interesting preliminary results. Nivolumab is being tested in a frontline setting versus sorafenib. At the same time, tremelimumab is under investigation in a second-line trial versus best supportive care.

- The potential role of immunotherapy in patients who cannot tolerate multikinase inhibitors, in those with more deteriorated liver function, and in the adjuvant setting makes this therapeutic approach of particular interest in HCC patients.

\section{References}

Papers of special note have been highlighted as: $\bullet$ of interest

1. Stotz M, Gerger A, Haybaeck J et al. Molecular targeted therapies in hepatocellular carcinoma: past, present and future. Anticancer Res. 35, 5737-5744 (2015).

2. Llovet JM, Ricci S, Mazzaferro V et al. Sorafenib in advanced hepatocellular carcinoma. N. Engl. J. Med. 359, 378-390 (2008).

- The first Phase III study showing the efficacy of sorafenib in hepatocellular carcinoma (HCC).

3. Cheng AL, Kang YK, Chen Z et al. Efficacy and safety of sorafenib in patients in the Asia-Pacific region with advanced hepatocellular carcinoma: a Phase III randomised, double-blind, placebo-controlled trial. Lancet Oncol. 10, 25-34 (2009).

4. Llovet JM, Decaens T, Raoul JL et al. Brivanib in patients with advanced hepatocellular carcinoma who were intolerant to sorafenib or for whom sorafenib failed: results from the randomized Phase III BRISK-PS study. J. Clin. Oncol. 3, 3509-3516 (2013).

5. Zhu AX, Park JO, Ryoo BY et al. Ramucirumab versus placebo as second-line treatment in patients with advanced hepatocellular carcinoma following first-line, therapy with sorafenib (REACH): a randomised, double-blind, multicentre, Phase III trial. Lancet Oncol. 16, 859-870 (2015).

6. Zhu AX, Kudo M, Assenat $\mathrm{E}$ et al. Effect of everolimus on survival in advanced hepatocellular carcinoma after failure of sorafenib: the EVOLVE-1 randomized clinical trial. JAMA 312, 57-67 (2014).

7. Abou-Alfa GK, Qin S, Ryoo BY et al. Phase III randomized study of second line ADI-peg 20 (A) plus best supportive care versus placebo (P) plus best supportive care in patients (pts) with advanced hepatocellular carcinoma (HCC). J. Clin. Oncol. 34(Suppl. 15) Abstract 4017 (2016).

8. Granito A, Guidetti E, Gramantieri L. c-MET receptor tyrosine kinase as a molecular target in advanced hepatocellular carcinoma. $J$. Hepatocell. Carcinoma 2, 29-38 (2015).

9. Santoro A, Simonelli M, Rodriguez-Lope C et al. A Phase-IB study of tivantinib (ARQ 197) in adult patients with hepatocellular carcinoma and cirrhosis. Br. J. Cancer 108, 21-24 (2013).

10. ArQule Press Release. http://investors.arqule.com

11. Bruix J, Qin S, Merle P et al. Regorafenib for patients with hepatocellular carcinoma who progressed on sorafenib treatment (RESORCE): a randomised, double-blind, placebo-controlled, Phase III trial. Lancet 389, 56-66 (2016).

- The first positive study in the second-line setting of HCC.

12. Cheng A-L, Finn RS, Qin S et al. Phase III trial of lenvatinib (LEN) vs sorafenib (SOR) in first-line treatment of patients (pts) with unresectable hepatocellular carcinoma (uHCC). J. Clin. Oncol. 35(Suppl. 15) 4001-4001 (2017).

13. Sangro B, Gomez-Martin C, de la Mata M et al. A clinical trial of CTLA-4 blockade with tremelimumab in patients with hepatocellular carcinoma and chronic hepatitis C. J. Hepatol. 59, 81-88 (2013).

14. El-Khoueiry AB, Sangro B, Yau T et al. Nivolumab in patients with advanced hepatocellular carcinoma (CheckMate 040): an open-label, non-comparative, Phase I/II dose escalation and expansion trial. Lancet 389(10088), 2492-2502 (2017).

15. Adnane L, Trail PA, Taylor I et al. Sorafenib (BAY 43-9006, Nexavar $\left.{ }^{\circledR}\right)$, a dual-action inhibitor that targets RAF/MEK/ERK pathway in tumor cells and tyrosine kinases VEGFR/PDGFR in tumor vasculature. Methods Enzymol. 407, 597-612 (2006). 
16. Wilhelm S, Carter C, Lynch M et al. Discovery and development of sorafenib: a multikinase inhibitor for treating cancer. Nat. Rev. Drug Discov. 5, 835-844 (2006).

17. Wilhelm SM, Carter C, Tang L et al. BAY 43-9006 exhibits broad spectrum oral antitumor activity and targets the RAF/MEK/ERK pathway and receptor tyrosine kinases involved in tumor progression and angiogenesis. Cancer Res. 64, 7099-7109 (2004).

18. Bruix J, Raoul JL, Sherman M et al. Efficacy and safety of sorafenib in patients with advanced hepatocellular carcinoma: subanalyses of a Phase III trial. J. Hepatol. 57, 821-829 (2012).

19. Bruix J, Cheng AL, Meinhardt G et al. Prognostic factors and predictors of sorafenib benefit in patients with hepatocellular carcinoma: analysis of two Phase III studies. J. Hepatol. 67, 999-1008 (2017).

20. Cheng AL, Guan Z, Chen Z et al. Efficacy and safety of sorafenib in patients with advanced hepatocellular carcinoma according to baseline status: subset analyses of the Phase III sorafenib Asia-Pacific trial. Eur. J. Cancer 48, 1452-1465 (2012).

21. Marrero JA, Kudo M, Venook AP et al. Observational registry of sorafenib use in clinical practice across Child-Pugh subgroups: the GIDEON study. J. Hepatol. 65, 1140-1147 (2016).

- The largest real-life study on sorafenib therapy in different subgroups of HCC patients.

22. Ganten TM, Stauber RE, Schott E et al. Sorafenib in patients with hepatocellular carcinoma-results of the observational INSIGHT Study. Clin. Cancer Res. 23, 5720-5728 (2017).

23. Iavarone M, Cabibbo G, Piscaglia F et al. Field-practice study of sorafenib therapy for hepatocellular carcinoma: a prospective multicenter study in Italy. Hepatology 54, 2055-2063 (2011).

24. Tovoli F, Negrini G, Bolondi L. Comparative analysis of current guidelines for the treatment of hepatocellular carcinoma. Hepat. Oncol. 3, 119-136 (2016).

25. Abou-Alfa GK, Amadori D, Santoro A et al. Safety and efficacy of sorafenib in patients with hepatocellular carcinoma (HCC) and Child-Pugh A versus B cirrhosis. Gastrointest. Cancer Res. 4, 40-44 (2011).

26. Furuse J, Ishii H, Nakachi K et al. Phase I study of sorafenib in Japanese patients with hepatocellular carcinoma. Cancer Sci. 99, 159-165 (2008).

27. Pinter M, Sieghart W, Hucke F et al. Prognostic factors in patients with advanced hepatocellular carcinoma treated with sorafenib. Aliment. Pharmacol. Ther. 34, 949-959 (2011).

28. Hollebecque A, Cattan S, Romano O et al. Safety and efficacy of sorafenib in hepatocellular carcinoma: the impact of the Child-Pugh score. Aliment. Pharmacol. Ther. 34, 1193-1201 (2011).

29. Wörns MA, Koch S, Niederle IM et al. The impact of patient and tumour baseline characteristics on the overall survival of patients with advanced hepatocellular carcinoma treated with sorafenib. Dig. Liver Dis. 45, 408-413 (2013).

30. Granito A, Bolondi L. Non-transplant therapies for patients with hepatocellular carcinoma and Child-Pugh-Turcotte class B cirrhosis. Lancet Oncol. 18, e101-e112 (2017).

31. Williet N, Clavel L, Bourmaud A et al. Tolerance and outcomes of sorafenib in elderly patients treated for advanced hepatocellular carcinoma. Dig. Liver Dis. 49, 1043-1049 (2017).

32. Brunot A, Le Sourd S, Pracht M et al. Hepatocellular carcinoma in elderly patients: challenges and solutions. J. Hepatocell. Carcinoma 17, 9-18 (2016).

33. Llovet JM, Peña CE, Bruix J et al. Plasma biomarkers as predictors of outcome in patients with advanced hepatocellular carcinoma. Clin. Cancer Res. 18, 2290-2300 (2012).

34. Shao YY, Hsu CH, Cheng AL. Predictive biomarkers of sorafenib efficacy in advanced hepatocellular carcinoma: are we getting there? World J. Gastroenterol. 21, 10336-10347 (2015).

35. Wang XW, Thorgeirsson SS. The biological and clinical challenge of liver cancer heterogeneity. Hepat. Oncol. 1, 349-353 (2014).

36. Howell JA, Sharma R. The clinical role of 'liquid biopsy' in hepatocellular carcinoma. Hepat. Oncol. 3, 45-55 (2016).

37. Howell J, Pinato DJ, Ramaswami R et al. On-target sorafenib toxicity predicts improved survival in hepatocellular carcinoma: a multi-centre, prospective study. Aliment. Pharmacol. Ther. 45, 1146-1155 (2017).

38. Di Costanzo GG, Casadei Gardini A, Marisi G et al. Validation of a simple scoring system to predict sorafenib effectiveness in patients with hepatocellular carcinoma. Target Oncol. 12(6), 795-803 (2017).

39. Reig M, Torres F, Rodriguez-Lope C et al. Early dermatologic adverse events predict better outcome in HCC patients treated with sorafenib. J. Hepatol. 61, 318-324 (2014).

40. Rimola J, Díaz-González Á, Darnell A et al. Complete response under sorafenib in patients with hepatocellular carcinoma. Relationship with dermatologic adverse. doi:10.1002/hep.29515 (2017) (Epub ahead of print).

41. Granito A, Marinelli S, Bolondi L et al. Prognostic significance of adverse events in patients with hepatocellular carcinoma treated with sorafenib. Therap. Adv. Gastroenterol. 9, 240-249 (2016).

42. Clare KE, Miller MH, Dillon JF. Editorial: sorafenib toxicity, a biomarker of effect? Aliment. Pharmacol. Ther. 45, 1469-1470 (2017). 
43. Boudou-Rouquette $\mathrm{P}$, Ropert $\mathrm{S}$, Mir O et al. Variability of sorafenib toxicity and exposure over time: a pharmacokinetic/pharmacodynamic analysis. Oncologist 17, 1204-1212 (2012).

44. Scartozzi M, Faloppi L, Svegliati-Baroni G et al. VEGF and VEGFR genotyping in the prediction of clinical outcome for HCC patients receiving sorafenib: the ALICE-1 study. Int. J. Cancer 135, 1247-1256 (2014).

45. Bruix J, Takayama T, Mazzaferro V et al. Adjuvant sorafenib for hepatocellular carcinoma after resection or ablation (STORM): a Phase III, randomised, double-blind, placebo-controlled trial. Lancet Oncol. 16, 1344-1354 (2015).

46. Lencioni R, Llovet JM, Han G et al. Sorafenib or placebo plus TACE with doxorubicin-eluting beads for intermediate stage HCC: the SPACE trial. J. Hepatol. 64, 1090-1098 (2016).

47. Sansonno D, Lauletta G, Russi S et al. Transarterial chemoembolization plus sorafenib: a sequential therapeutic scheme for HCV-related intermediate-stage hepatocellular carcinoma: a randomized clinical trial. Oncologist 17, 359-366 (2012).

48. Kudo M, Imanaka K, Chida N et al. Phase III study of sorafenib after transarterial chemoembolisation in Japanese and Korean patients with unresectable hepatocellular carcinoma. Eur. J. Cancer 47, 2117-2127 (2011).

49. Galle PR, Tovoli F, Foerster F et al. The treatment of intermediate stage tumours beyond TACE: from surgery to systemic therapy. J. Hepatol. 67, 173-183 (2017).

- A comprehensive review dealing with eterogeneity of patients with intermediate stage of HCC and their treatment options.

50. Brade AM, $\mathrm{Ng} \mathrm{S}$, Brierley J et al. Phase I Trial of sorafenib and stereotactic body radiation therapy for hepatocellular carcinoma. Int. J. Radiat. Oncol. Biol. Phys. 94, 580-587 (2016).

51. ClinicalTrials.gov. Identifier NCT01556490. https://clinicaltrials.gov/ct2/show/NCT01556490

52. ClinicalTrials.gov. Identifier NCT01126645. https://clinicaltrials.gov/ct2/show/NCT01126645

53. Shojaei F. Anti-angiogenesis therapy in cancer: current challenges and future perspectives. Cancer Lett. 320, 130-137 (2012).

54. Ray EM, Sanoff HK. Optimal therapy for patients with hepatocellular carcinoma and resistance or intolerance to sorafenib: challenges and solutions. J. Hepatocell. Carcinoma 4, 131-138 (2017).

55. Reig M, Rimola J, Torres F et al. Postprogression survival of patients with advanced hepatocellular carcinoma: rationale for second-line trial design. Hepatology 58, 2023-2031 (2013).

- The first study demonstrating the different prognosis of patients after sorafenib failure according to the progression pattern.

56. Iavarone M, Cabibbo G, Biolato $\mathrm{M}$ et al. Predictors of survival in patients with advanced hepatocellular carcinoma who permanently discontinued sorafenib. Hepatology 62, 784-791 (2015).

57. Tovoli F, De Lorenzo S, Barbera MA et al. Postsorafenib systemic treatments for hepatocellular carcinoma: questions and opportunities after the regorafenib trial. Future Oncol. 13, 1893-1905 (2017).

58. Kuo YH, Wang JH, Hung CH et al. Albumin-bilirubin grade predicts prognosis of HCC patients with sorafenib use. J. Gastroenterol. Hepatol. 32, 1975-1981 (2017).

59. Pinato DJ, Yen C, Bettinger $\mathrm{D}$ et al. The albumin-bilirubin grade improves hepatic reserve estimation post-sorafenib failure: implications for drug development. Aliment. Pharmacol. Ther. 45, 714-722 (2017).

60. Edeline J, Blanc JF, Campillo-Gimenez B et al. Prognostic scores for sorafenib-treated hepatocellular carcinoma patients: a new application for the hepatoma arterial embolisation prognostic score. Eur. J. Cancer 86, 135-142 (2017).

61. Matsui J, Yamamoto Y, Funahashi Y et al. E7080, a novel inhibitor that targets multiple kinases, has potent antitumor activities against stem cell factor producing human small cell lung cancer H146, based on angiogenesis inhibition. Int. J. Cancer 122, 664-671 (2008).

62. Schlumberger M, Tahara M, Wirth LJ et al. Lenvatinib versus placebo in radioiodine-refractory thyroid cancer. N. Engl. J. Med. 372, 621-630 (2015).

63. Choueiri TK, Escudier B, Powles T et al. Cabozantinib versus everolimus in advanced renal cell carcinoma (METEOR): final results from a randomised, open-label, Phase III trial. Lancet Oncol. 17, 917-927 (2016).

64. Wilhelm SM, Dumas J, Adnane L et al. Regorafenib (BAY 73-4506): a new oral multikinase inhibitor of angiogenic, stromal and oncogenic receptor tyrosine kinases with potent preclinical antitumor activity. Int. J. Cancer 129, 245-255 (2011).

65. Wilhelm SM, Carter C, Tang L et al. BAY 43-9006 exhibits broad spectrum oral antitumor activity and targets the RAF/MEK/ERK pathway and receptor tyrosine kinases involved in tumor progression and angiogenesis. Cancer Res. 64, 7099-7109 (2004).

66. Tsai JH, Lee WMF. Tie2 in tumor endothelial signaling and survival: implications for antiangiogenic therapy. Mol. Cancer Res. 7 , 300-310 (2009).

67. Bruix J, Merle P, Granito A et al. Efficacy and safety of regorafenib versus placebo in patients with hepatocellular carcinoma (HCC) progressing on sorafenib: results of the international, randomized Phase III RESORCE trial (abstract LBA-03). Ann. Oncol. 27(Suppl. 2), ii140-ii141 (2016).

68. Krishnamoorthy SK, Relias V, Sebastian S et al. Management of regorafenib-related toxicities: a review. Therap. Adv. Gastroenterol. 8, 285-297 (2015). 
69. Merle P, Granito A, Huang $\mathrm{YH}$ et al. Time course of treatment-emergent adverse events (TEAEs) in the randomized, controlled Phase III RESORCE trial of regorafenib for patients with hepatocellular carcinoma. Hepatology 66, 726A-726A (2017).

70. Finn RS, Merle P, Granito A et al. Outcomes with sorafenib (SOR) followed by regorafenib (REG) or placebo (PBO) for hepatocellular carcinoma (HCC): results of the international, randomized Phase III RESORCE trial. J. Clin. Oncol. 35(Suppl. 4S), Abstract 344 (2017).

71. Ciamporcero E, Miles KM, Adelaiye R et al. Combination strategy targeting VEGF and HGF/c-met in human renal cell carcinoma models. Mol. Cancer Ther. 14, 101-110 (2015).

72. Daudigeos-Dubus E, Le Dret L, Bawa O et al. Dual inhibition using cabozantinib overcomes HGF/MET signaling mediated resistance to pan-VEGFR inhibition in orthotopic and metastatic neuroblastoma tumors. Int. J. Oncol. 50, 203-211 (2017).

73. Sennino B, Ishiguro-Oonuma T, Wei Y et al. Suppression of tumor invasion and metastasis by concurrent inhibition of c-Met and VEGF signaling in pancreatic neuroendocrine tumors. Cancer Discov. 2, 270-287 (2012).

74. You H, Ding W, Dang H et al. c-Met represents a potential therapeutic target for personalized treatment in hepatocellular carcinoma. Hepatology 54, 879-889 (2011).

75. Rimassa L, Abbadessa G, Personeni N et al. Tumor and circulating biomarkers in patients with second-line hepatocellular carcinoma from the randomized Phase II study with tivantinib. Oncotargets 7, 72622-72633 (2016).

76. Kelley RK, Verslype C, Cohn AL et al. Cabozantinib in hepatocellular carcinoma: results of a Phase II placebo-controlled randomized discontinuation study. Ann. Oncol. 28, 528-534 (2017).

77. Abou-Alfa GK, Cheng AI, Meyer T et al. Phase III randomized, double-blind, controlled study of cabozantinib (XL184) versus placebo in subjects with hepatocellular carcinoma who have received prior sorafenib (CELESTIAL; NCT01908426). J. Clin. Oncol. 32(Suppl. 5) Abstract TPS4150 (2014).

78. EXELIXIS. http://ir.exelixis.com/phoenix.zhtml?c=120923\&p=irol-newsArticle\&ID=2318244

79. Forner A, Reig M. Does ramucirumab deserve a second chance for liver cancer? Lancet Oncol. 16, 751-752 (2015).

80. Kim HY, Park JW. Current immunotherapeutic strategies in hepatocellular carcinoma: recent advances and future directions. Therap. Adv. Gastroenterol. 10, 805-814 (2017).

- An exhaustive review of immunotherapeutic approach of HCC.

81. Lesokhin AM, Callahan MK, Postow MA et al. On being less tolerant: enhanced cancer immunosurveillance enabled by targeting checkpoints and agonists of T cell activation. Sci. Transl. Med. 7, 280sr1 (2015).

82. Mittal D, Gubin MM, Schreiber RD et al. New insights into cancer immunoediting and its three component phases-elimination, equilibrium and escape. Curr. Opin. Immunol. 27, 16-25 (2014).

83. Sangro B, Gomez-Martin C, de la Mata M et al. A clinical trial of CTLA-4 blockade with tremelimumab in patients with hepatocellular carcinoma and chronic hepatitis C. J. Hepatol. 59, 81-88 (2013).

84. Duffy AG, Ulahannan SV, Makorova-Rusher $\mathrm{O}$ et al. Tremelimumab in combination with ablation in patients with advanced hepatocellular carcinoma. J. Hepatol. 66, 545-551 (2017).

85. ClinicalTrials.gov. Identifier NCT01658878. https://clinicaltrials.gov/ct2/show/NCT01658878

86. Kudo M. Immune checkpoint inhibition in hepatocellular carcinoma: basics and ongoing clinical trials. Oncology 92(Suppl. 1), 50-62 (2017).

87. El-Khoueiry AB, Sangro B, Yau T et al. Nivolumab in patients with advanced hepatocellular carcinoma (CheckMate 040): an open-label, non-comparative, Phase I/II dose escalation and expansion trial. Lancet 389, 2492-2502 (2017).

88. Sangro B, Park JW, Dela Cruz CM et al. A randomized, multicenter, Phase III study of nivolumab vs sorafenib as first-line treatment in patients (pts) with advanced hepatocellular carcinoma (HCC): CheckMate-459. J. Clin. Oncol. 34(Suppl. 15) Abstract TPS4147 (2016).

89. Finn RS, Chan SL, Zhu AX et al. KEYNOTE-240: randomized Phase III study of pembrolizumab versus best supportive care for second-line advanced hepatocellular carcinoma. J. Clin. Oncol. 35(Suppl. 4S), Abstract TPS503 (2017).

90. Kareva I, Waxman DJ, Lakka Klement G. Metronomic chemotherapy: an attractive alternative to maximum tolerated dose therapy that can activate anti-tumor immunity and minimize therapeutic resistance. Cancer Lett. 358, 100-106 (2015).

91. Pasquier E, Kavallaris M, André N. Metronomic chemotherapy: new rationale for new directions. Nat. Rev. Clin. Oncol. 7, 455-465 (2010).

92. Granito A, Marinelli S, Terzi E et al. Metronomic capecitabine as second-line treatment in hepatocellular carcinoma after sorafenib failure. Dig. Liver Dis. 47, 518-522 (2015).

93. Brandi G, de Rosa F, Agostini V et al. Metronomic capecitabine in advanced hepatocellular carcinoma patients: a Phase II study. Oncologist 18, 1256-1257 (2013).

94. Casadei Gardini A, Foca F, Scartozzi M et al. Metronomic capecitabine versus best supportive care as second-line treatment in hepatocellular carcinoma: a retrospective study. Sci. Rep. 7, 42499 (2017).

95. Marinelli S, Granito A, Piscaglia F et al. Metronomic capecitabine in patients with hepatocellular carcinoma unresponsive to or ineligible for sorafenib treatment: report of two cases. Hepat. Mon. 13, e11721 (2013). 
96. A multicenter, randomized, open-label Phase III study of two anti-angiogenic strategies in advanced hepatocellular carcinoma patients with cross-over at first-line failure: metronomic Capecitabine/Sorafenib (Arm A) vs Sorafenib/metronomic Capecitabine (Arm B). www.agenziaf armaco.gov.it/sites/default/files/LEGENDA_GRADUATORIA_DEFINITIVA_85_protocolli.pdf 
